\title{
Operando NMR of NMC811/graphite lithium-ion batteries: Structure, dynamics, and lithium metal deposition
}

\author{
Katharina Märker, Chao Xu, Clare P. Grey* \\ Department of Chemistry, University of Cambridge, Lensfield Road, Cambridge CB2 1EW, UK \\ The Faraday Institution, Quad One, Harwell Science and Innovation Campus, Didcot OX11 ORA, UK
}

\begin{abstract}
Lithium-ion batteries (LIBs) are of tremendous importance for our society, but their limited lifetime still poses a great challenge. For a better understanding of battery cycling and degradation, operando analytical measurements are invaluable. In this work, we demonstrate that operando $^{7} \mathrm{Li}$ nuclear magnetic resonance (NMR) spectroscopy can be applied to full LIBs. We exemplify this on $\mathrm{LiNi}_{0.8} \mathrm{Mn}_{0.1} \mathrm{Co}_{0.1} \mathrm{O}_{2}$ (NMC811) / graphite cells, which are typical high-energy LIBs. Employing industry-standard electrodes, our operando cells show realistic cycling performance at practical rates, which allows us to conduct experiments at different rates and temperatures and to draw conclusions on the performance of LIBs. The NMR experiments monitor processes in both electrodes individually, including Li-ion mobility and its changes with temperature. Moreover, Li metal deposition on graphite is observed at low temperature, which is an important degradation mechanism in LIBs and a severe safety hazard. Our experiments offer unique insights into this Li metal deposition process under different charging conditions.
\end{abstract}

\section{INTRODUCTION}

Li-ion batteries (LIBs) are ubiquitous in our daily lives, with applications ranging from small electronic devices to electric vehicles. They are attractive energy storage solutions due to their high energy density, however, their lifetime is limited by gradual capacity loss or even full failure. ${ }^{1-3}$ It is often not trivial to pinpoint the origin of this performance loss, let alone predict the cycle life of a cell. Therefore, a more holistic understanding of physical, chemical, and electrochemical processes in a functioning LIB is required. In order to develop this understanding, it is crucial to conduct analytical measurements operando on a working battery. ${ }^{4}$

Operando measurements on batteries allow monitoring different states-of-charge (SOC) repeatedly, in principle throughout their whole cycle lives, providing access to a wealth of analytical data compared to a single ex situ or post mortem analysis. Moreover, transient processes may be observed which may no longer be detectable in ex situ measurements on a relaxed electrode. However, most operando techniques face great technical challenges in cell design and experimental setup, which often leads to practical limitations. ${ }^{4}$ For instance, only slow charging rates can be applied and/or "half-cells" are used where one electrode is replaced by Li metal. While such measurements are very valuable for answering specific questions, they are less suitable for studying the performance and degradation mechanisms of batteries in practical configurations and under realistic cycling conditions.

Nuclear magnetic resonance (NMR) spectroscopy is wellsuited for operando measurements on electrochemical cells as it is a non-invasive, non-destructive technique which is sensitive to both structure and dynamics. NMR experiments on LIBs benefit from a comparably high sensitivity since ${ }^{7} \mathrm{Li}(\operatorname{spin}-3 / 2)$ possesses a high natural abundance $(92.4 \%)$, high gyromagnetic ratio $\left(\gamma\left({ }^{7} \mathrm{Li}\right) / \gamma\left({ }^{1} \mathrm{H}\right)=0.39\right)$ and usually experiences only small quadrupolar couplings. Considerable technical advances have been made in in situ and operando NMR in the past years through the design of dedicated electrochemical cells and NMR probes, ${ }^{5-8}$ some of which now facilitate the advanced studies presented in this paper. Note that we refer to operando measurements as being performed during electrochemical cycling, while in situ measurements are also performed on intact cells, but not necessarily at the same time as charge and discharge.

A typical LIB is assembled with a graphite anode and a layered transition metal (TM) oxide cathode. In both, $\mathrm{Li}$ ions reside in layers between the graphene sheets or the TM oxide layers, respectively, and are reversibly inserted and extracted during electrochemical cycling. As cathode materials, Ni-rich mixed TM oxides are currently of great interest for automotive applications owing to their high specific capacity and low concentration of the expensive, scarce and toxic cobalt. ${ }^{9} 10$ One representative composition is $\mathrm{LiNi}_{0.8} \mathrm{Mn}_{0.1} \mathrm{Co}_{0.1} \mathrm{O}_{2}$ (NMC811) which delivers a high capacity at comparably low voltage. ${ }^{11}$ However, this comes at the expense of faster degradation compared to cathode materials with lower nickel content. ${ }^{11}$

Li-ion transport kinetics in the electrode materials play a key role for battery performance. In both graphite anodes and layered TM oxide cathodes, two-dimensional Li diffusion dominates where $\mathrm{Li}$ ions hop between lattice positions within their respective layers. ${ }^{12,13}$ This diffusion is often a major limiting factor for a battery's rate capability, with insufficient Li mobility leading to overpotentials and hence a lower reversible capacity within a given potential range. For fast charging applications, fast $\mathrm{Li}$-ion diffusion is therefore essential.

Another critical degradation process occurs when Li metal deposits on the surface of the graphite anode instead of intercalating into the material. ${ }^{14-16}$ This Li metal formation is not thermodynamically favoured, but is promoted by low temperatures, fast charging rates, and charge to high potentials. Under such conditions, reduction to Li metal can become the kinetically favoured process as several factors hinder $\mathrm{Li}$ intercalation into graphite. Firstly, the charge-transfer barrier associated with $\mathrm{Li}$ transport through the SEI is harder to overcome at low 
temperature. ${ }^{16}$ Secondly, Li diffusivity in graphite slows down with decreasing temperature and with increasing SOC of the material. ${ }^{12}$ Slow Li diffusion leads to the buildup of concentration gradients between the surface and the bulk of graphite particles during charge. If the graphite surface is saturated, no more $\mathrm{Li}$ ions can intercalate and reduction to $\mathrm{Li}$ metal will occur instead. Finally, Li diffusion in the electrolyte also slows down with decreasing temperature and contributes to increasing cell polarization. However, it is usually not considered to be the dominant influence on Li metal deposition. ${ }^{17}$ Extensive and prolonged Li plating poses a serious safety hazard as it can lead to short circuits and thermal runaway. But even without such severe consequences, capacity fade is significantly accelerated as new solid electrolyte interphase (SEI) is formed continuously on fresh $\mathrm{Li}$ metal surfaces, consuming electrolyte and active $\mathrm{Li}$ and leading to increased resistance. Additionally, Li metal can lose electrical contact to the anode during discharge and remain inactive in the cell as so-called "dead lithium". Magnetic resonance techniques are valuable tools for investigating Li metal deposition, and in situ and operando NMR measurements have been instrumental in studying this process in Li metal batteries. ${ }^{18-20}$ Fewer applications have been made to Li metal deposition on graphite at low temperature: Wandt et al. have reported operando measurements using electron paramagnetic resonance $^{21}$ and Arai et al. employed in situ NMR at room temperature to detect deposited Li metal after low-temperature cycling. ${ }^{22}$ To the best of our knowledge no operando NMR of $\mathrm{Li}$ metal deposition on graphite in full-cells at low temperatures has been reported so far.

In this work, we report operando ${ }^{7} \mathrm{Li}$ NMR measurements of NMC811/graphite full-cells with electrochemical performance comparable to that of coin cells. We present data recorded at temperatures between -20 and $+55^{\circ} \mathrm{C}$, based on which we investigate cell performance and degradation processes. We examine Li dynamics and their changes with temperature in both electrodes, as well as Li plating and stripping processes at low temperature. Moreover, we present measurements on a cell with poor electrochemical performance and identify related spectroscopic signatures. To our knowledge, this is the first operando NMR study of a full LIB in which both electrodes are investigated simultaneously. We provide guidance on how to perform and interpret measurements on such a complex and heterogeneous system, including the investigation of the highly paramagnetic NMC811 cathode. The methodology presented here can be applied for studying related LIB systems and lays the basis for investigating numerous phenomena during battery cycling.

\section{MATERIALS AND METHODS}

Materials. Coated NMC811 and graphite electrodes were fabricated by the Cell Analysis, Modeling, and Prototyping (CAMP) Facility at the Argonne National Laboratory. The NMC cathodes (batch code A-C020) consist of $90 \mathrm{wt} \%$ NMC811 (Targray), $5 \mathrm{wt} \%$ polyvinylidene fluoride (PVDF) binder (Solvay 5130), and 5 wt\% carbon black (Timcal C45). The electrodes have a thickness of $53 \mu \mathrm{m}$ (including $20 \mu \mathrm{m} \mathrm{Al}$ foil), a porosity of $32.5 \%$ and an areal loading of $9.12 \mathrm{mg} / \mathrm{cm}^{2}$. The graphite electrodes are composed of $91.83 \mathrm{wt} \%$ graphite powder (Hitachi MagE3), 2 wt\% carbon black (Timcal C45), 6 wt\% PVDF binder (Kureha 9300), and 0.17 wt\% oxalic acid. The electrodes have a thickness of $52 \mu \mathrm{m}$ (including $10 \mu \mathrm{m} \mathrm{Cu}$ foil), a porosity of $30.3 \%$ and an areal loading of $6.35 \mathrm{mg} / \mathrm{cm}^{2}$. The N/P ratio (capacity ratio of the negative to positive electrode) is $\sim 1.28$, estimated based on a practical capacity of
$200 \mathrm{mAh} / \mathrm{g}$ for NMC811 and $360 \mathrm{mAh} / \mathrm{g}$ for graphite. These electrodes are referred to as "coated electrodes" in this manuscript and were used for all experiments, unless stated otherwise (see also Table S1). Details on other electrodes used in this work can be found in the SI, section 1 .

For operando NMR experiments, the electrodes were cut into rectangular shape with dimensions of $1.3 \times 0.6 \mathrm{~cm}$ for NMC811 and $1.4 \times 0.6 \mathrm{~cm}$ for graphite. They were then dried at $120^{\circ} \mathrm{C}$ for $12 \mathrm{~h}$ under dynamic vacuum in a Büchi oven before being transferred to a glovebox. A $1 \mathrm{M}$ solution of $\mathrm{LiPF}_{6}$ in ethylene carbonate / ethyl methyl carbonate 3:7 v/v (LP57, SoulBrain MI, USA) was used as the electrolyte. Glass fibre separator was dried at $100{ }^{\circ} \mathrm{C}$ in a vacuum oven over several days before use.

In situ NMR cell assembly. Capsule cells fabricated from polyether ether ketone (PEEK) were used in all experiments, as described by Pecher et al. ${ }^{5}$ They were assembled in an Ar-filled glovebox $\left(\mathrm{H}_{2} \mathrm{O}\right.$ and $\mathrm{O}_{2}$ levels below $\left.1 \mathrm{ppm}\right)$ using an NMC811 cathode, a graphite anode, one glass fibre separator and LP57 electrolyte. See SI section 1 for more details.

Electrochemical cycling. For all cells shown in this work, two formation cycles at $\mathrm{C} / 15$ were conducted at room temperature prior to cycling at higher rates. C-rates were calculated based on a reversible capacity of $200 \mathrm{mAh} / \mathrm{g}$ of NMC811. All cells were cycled between 4.3 and $2.5 \mathrm{~V}$. Constant current (CC) charge and discharge was mostly employed at room temperature, whereas constant current - constant voltage (CCCV) protocols were used at lower temperatures. The voltage was held during $\mathrm{CV}$ periods until the current dropped below C/15. A voltage hold at $2.5 \mathrm{~V}$ was performed when the operating temperature was changed in order to start the following cycle from a fully discharged state. Charging rates, protocols and temperatures are given in the respective Figure captions and more details can be found in Table S1.

Operando NMR experiments. All NMR experiments were performed on a $7.05 \mathrm{~T}$ (117 MHz ${ }^{7} \mathrm{Li}$ Larmor frequency) Bruker Avance NMR spectrometer. A dedicated in situ NMR probe (NMR Service $\mathrm{GmbH}$ ) was employed. It possesses automatic tuning and matching capabilities, built-in highly shielded electrochemistry connections and variable temperature (VT) capabilites. ${ }^{23}$ Additional radio frequency (RF) low-pass filters were used in the connection to the potentiostat. A solenoid coil of $12 \mathrm{~mm}$ inner diameter was used for all experiments and cells were oriented such that the electrodes were parallel to the main magnetic field because this yielded the largest separation between the NMC811 and the electrolyte signal (see Figure S16). Temperature control was achieved with a dry nitrogen gas flow which was heated using the heat element in the probe or cooled using Bruker BCU-II or BCU-05 cooling units. The temperatures given in this work refer to the reading of a temperature sensor close to the in situ cell.

During electrochemical cycling, NMR spectra were acquired continuously, using a pulse sequence as illustrated in Figure S2. First, the probe tuning was adjusted automatically. ${ }^{23}$ Then, ${ }^{7} \mathrm{Li}$ NMR spectra were acquired, one after another, for Li ions in NMC811 ("NMC spectra"), in graphite ("graphite spectra") and, for the datasets in Figures 3 and S10-13, in Li metal ("Li metal spectra"). ${ }^{7} \mathrm{Li}$ hard pulses were applied at RF-field strengths of $\sim 50 \mathrm{kHz}$. For chemical shift referencing, the maximum of the electrolyte signal was set to $0 \mathrm{ppm}$. No external chemical reference was employed because the chemical shifts of all components in the NMC/graphite in situ cells strongly depend on cell orientation (Figure S16). All data was recorded and 
processed using Bruker Topspin 2.1 and 3.6.2. Data analysis and plotting were carried out using home-written MATLAB scripts.

NMC811 spectra were recorded using variable offset cumulative spectroscopy (VOCS) ${ }^{24}$ Each spectrum was acquired using a solid echo sequence with echo delays of $5 \mu$ s and a recycle delay of $50 \mathrm{~ms}$. Graphite spectra were acquired using a onepulse sequence and a recycle delay of $20 \mathrm{~s}$. Li metal spectra were acquired using a one-pulse sequence and recycle delays of $0.2 \mathrm{~s}$ or $1 \mathrm{~s}$. The experimental times were as follows: $\sim 3 \mathrm{~min}$ $30 \mathrm{~s}$ for NMC spectra, $\sim 2 \mathrm{~min} 40 \mathrm{sec}$ for graphite spectra, $\sim 3 \mathrm{~min} 30 \mathrm{~s}$ for Li metal spectra. More details on the NMR experiments can be found in the SI, section 1 .

\section{RESULTS AND DISCUSSION}

Operando NMR of NMC811/graphite cells at room temperature. In situ NMR cells were assembled using capsule cells made from PEEK, ${ }^{5}$ employing NMC811 cathodes, graphite anodes, and LP57 electrolyte. All experiments used high-quality electrodes coated on metal current collector foils (see Materials and Methods for details), referred to as coated electrodes in the following, which enables us to apply realistic cycling rates.

Separate monitoring of $\mathrm{Li}$ ions in anode and cathode was achieved based on the very different spin-lattice $\left(T_{1}\right)$ relaxation times of $\mathrm{Li}$ ions in NMC811 and graphite. NMC811 has very short ${ }^{7} \mathrm{Li} T_{1}$ relaxation times of less than $10 \mathrm{~ms}$ due to the presence of paramagnetic TM ions $\left(\mathrm{Ni}^{2+}, \mathrm{Ni}^{3+}, \mathrm{Mn}^{4+}\right),{ }^{25}$ whereas $T_{1}$ relaxation times in $\mathrm{Li}_{\mathrm{x}} \mathrm{C}_{6}$ lie between 1 and $4 \mathrm{~s} .{ }^{26}$ Therefore, recycle delays of $50 \mathrm{~ms}$ and $20 \mathrm{~s}$ were employed for acquiring quantitative spectra of $\mathrm{Li}$ ions intercalated in the cathode ("NMC spectra") and the anode ("graphite spectra"), respectively. Signals from the respective other electrode were largely filtered out either due to their longer $\mathrm{T}_{1}$ relaxation time (graphite) or their very broad linewidths (NMC, see further explanation in SI section 1). The NMC and graphite spectra took $\sim 3$ minutes each to acquire, giving the operando measurements a time-resolution of $\sim 6$ minutes.

Figure 1a shows a typical ${ }^{7} \mathrm{Li}$ operando NMR dataset for a NMC811/graphite full-cell, recorded during the first two cycles (formation cycles) of the fresh cell. The dataset contains the voltage curve (left), as well as contour plots of the NMC (middle) and graphite (right) spectra. The voltage curve contains all expected features of a NMC811/graphite full cell as demonstrated by comparison to a coin cell made with the same electrodes and electrolyte (Figure S3a,c). The first-cycle coulombic efficiency was $84.8 \%$ which again is in good agreement with values obtained in coin cells. Figure 1b shows representative spectra of NMC811 and graphite obtained during the second charge of the cell, extracted to perform a more detailed analysis of the operando spectra (see Figure S5 for comparison to spectra acquired during the first charge). In all spectra, an intense signal from $\mathrm{Li}$ ions in the electrolyte is observed at $\sim 0 \mathrm{ppm}$ while the remaining signals correspond to $\mathrm{Li}$ ions in the respective electrodes.

The ${ }^{7} \mathrm{Li}$ NMR signal of lithiated NMC811 (Figure 1b, $2.7 \mathrm{~V}$ ) is very broad due to the presence of several paramagnetic TM ion species and the large number of different $\mathrm{Li}$ environments. $^{25,27}$ As the cell is charged, Li ions are extracted from NMC811 and its signal intensity decreases. Note that this decrease is not linear, which will be discussed in more detail below. The centre of the signal shifts towards $0 \mathrm{ppm}$ during charge due to the oxidation of $\mathrm{Ni}^{2+}$ and $\mathrm{Ni}^{3+}$ to diamagnetic $\mathrm{Ni}^{4+} .{ }^{25} \mathrm{At}$ 4.3 V when most Li has been extracted from NMC (Figure 1b, top blue spectrum), only a minor signal remains and the spectrum is dominated by the electrolyte peak.

$\mathrm{Li}$ intercalates into graphite in several stages, labelled as stages $4-1$, with the number referring to the number of graphene sheets between Li layers. ${ }^{28,29}$ The ${ }^{7} \mathrm{Li}$ NMR signal of the intercalated ions changes for each stage and one can broadly distinguish between dilute stages (stages 4 and $3 ; \mathrm{LiC}_{36}$ to $\mathrm{LiC}_{18}$ ) and dense stages (stages 2 and $1 ; \mathrm{LiC}_{12}, \mathrm{LiC}_{6}$ ), exhibiting signals between 0 and $15 \mathrm{ppm}$, and between 40 and $50 \mathrm{ppm}$, respectively. ${ }^{26,30,31}$ Our operando NMR spectra acquired on graphite (Figure 1b, red spectra) reflect these trends: first, signals from the dilute stages appear as a shoulder of the electrolyte peak at higher ppm values, followed by signals coming from the dense stages appearing between 45 and $50 \mathrm{ppm}$ while the signal intensity of the dilute stages decreases. We also observe additional weak signals in the dense stage spectra (asterisks in Figure 1b) which originate from the satellite transitions of the quadrupolar ${ }^{7} \mathrm{Li}$ nucleus. Since the quadrupolar coupling constants $\left(C_{\mathrm{Q}}\right)$ for stage 2 and 1 differ significantly $(\sim 34 \mathrm{kHz}$ for $\mathrm{LiC}_{12}$ and $\sim 45 \mathrm{kHz}$ for $\left.\mathrm{LiC}_{6}{ }^{26,30,31}\right)$, the satellite transition peaks can be used to distinguish the two stages: the graphite spectrum at $4.0 \mathrm{~V}$ originates mostly from $\mathrm{LiC}_{12}$, whereas $\mathrm{LiC}_{12}$ and $\mathrm{LiC}_{6}$ co-exist at the top of charge $(4.3 \mathrm{~V})$.

The patterns of changes in the NMC and graphite spectra repeat during charge and discharge in both cycles shown, demonstrating that the in situ cell cycles well over several days and that the lithiation and delithiation processes in both materials proceed in similar ways at slow rates. 

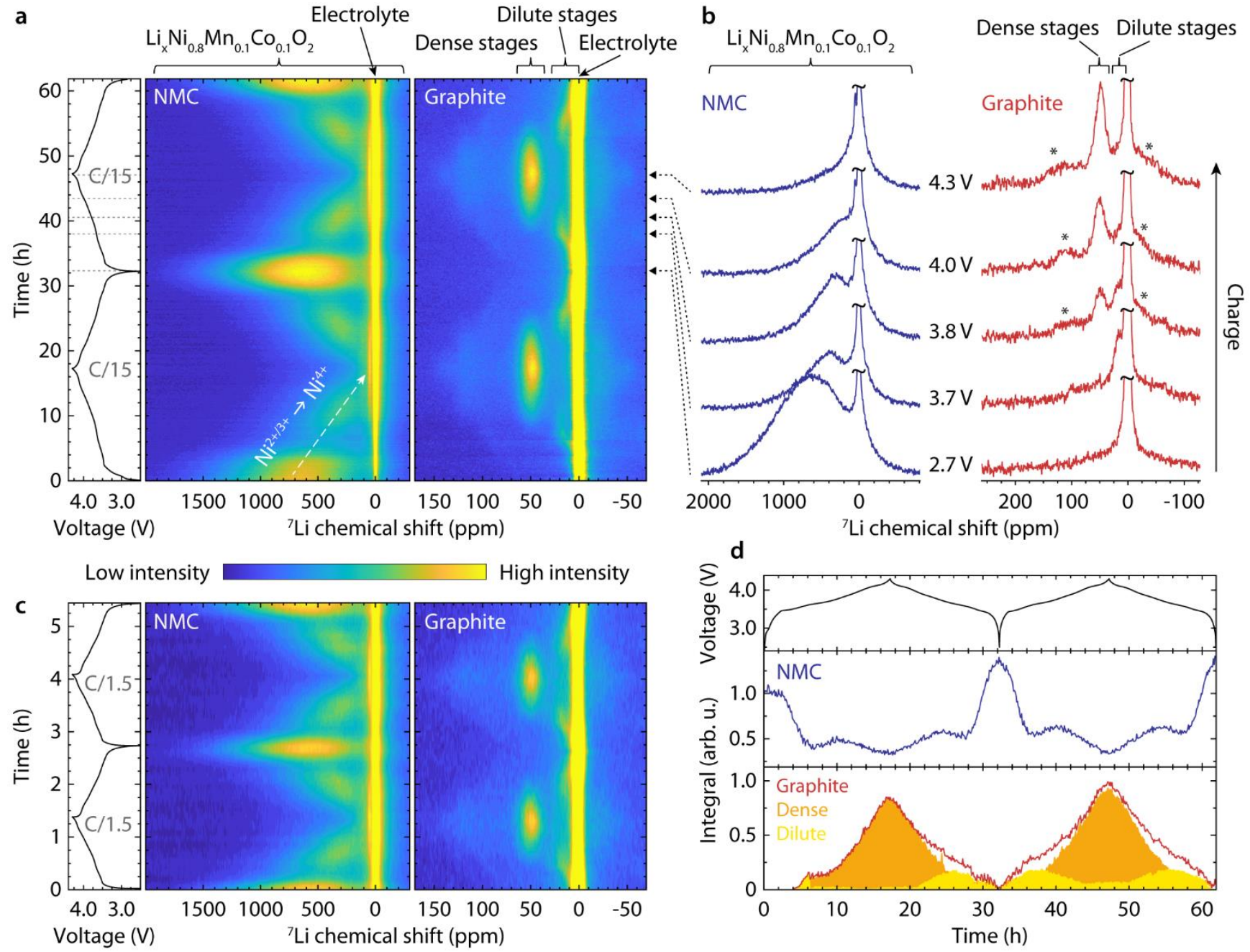

Figure 1. (a,c) Voltage profile and contour plots of operando ${ }^{7} \mathrm{Li}$ NMR data recorded on a NMC811/graphite cell during galvanostatic cycling between 4.3 and $2.5 \mathrm{~V}$ at $25^{\circ} \mathrm{C}$. The two formation cycles at $\mathrm{C} / 15$ and two later cycles at $\mathrm{C} / 1.5$ are shown in (a) and (c), respectively. (b) Representative NMC (blue) and graphite (red) spectra taken from the contour plots in (a) as indicated by the dotted lines. The same scaling has been applied for all NMC and graphite spectra, respectively, and the electrolyte signal at $\sim 0$ ppm has been truncated for clarity. A signal from dense stage graphite can be observed in the NMC spectra at high SOC, and asterisks in the graphite spectra indicate the satellite transitions. (d) Evolution of integrated signal intensities of NMC (blue) and graphite (red) during the two formation cycles obtained by deconvoluting the spectra in (a). The full graphite integrals are the sum of integrals from the dilute (yellow) and dense (orange) stages. Arbitrary scaling has been applied to the NMC and graphite integrals.

Figure 1d shows the integrated signal intensities for NMC (blue) and graphite (red) spectra, with the graphite further separated into the dilute (yellow) and dense (orange) stages. An increase of the overall signal intensity occurs during the first formation cycle, visible for instance when comparing the NMC integrals before electrochemical cycling $(t=0 \mathrm{~h})$ and after the first cycle $(t \approx 32 \mathrm{~h})$. We observed this signal increase over the first formation cycle in several NMC811/graphite cells and mainly attribute it to increasing probe sensitivity (further discussion in the SI, section 3). Apart from this trend, a linear change of the integrated ${ }^{7} \mathrm{Li}$ signal intensity would be expected because constant charge and discharge currents are applied. This is indeed the case for the summed lithiated graphite signal in Figure 1d (red), demonstrating that Li ions intercalated in graphite can be detected nearly quantitatively.

A strong deviation from linear behaviour is observed in the NMC integrals. The signal intensity decreases drastically at the beginning of charge before it partially recovers, the signal then almost completely disappears as more Li is removed from the structure. Similar trends have been observed in operando NMR studies of a $\mathrm{Li}$ excess spinel cathode, $\mathrm{Li}_{1.08} \mathrm{Mn}_{1.92} \mathrm{O}_{4}{ }^{32}$ They are caused by changes of Li-ion mobility and their influence on spin-spin relaxation times $T_{2}$, i.e. the relaxation time measured with an echo sequence. ${ }^{32} T_{2}$ ' relaxation times drop considerably when the Li hopping frequency is on the order of the frequency separation between ${ }^{7} \mathrm{Li}$ resonances (intermediate hopping regime, tens of $\mathrm{kHz}$ in our case). This causes a significant portion of the NMR signal to decay during the echo evolution, and the measured signal intensity is thus lower than expected. When $\mathrm{Li}$ hopping is within the fast or slow-motion regime, $T_{2}$ ' is considerably longer and less signal decays during the echo periods. Hence, the local minima in the integration curve during charge ( $t \approx 7 \mathrm{~h}$ and $37 \mathrm{~h}, \sim 35 \%$ SOC) correspond to an intermediate Li-ion hopping regime and signify the transition from slow to a fast $\mathrm{Li}$ motion. This interpretation will be supported by VT NMR measurements in the next section, and correlates well with our previous magic-angle spinning (MAS) NMR results on NMC811 where we observed a strong increase of Li-ion 
mobility from $\sim 20 \%$ SOC, which was also associated with a drop of $T_{2}$ ' in the NMR experiments. ${ }^{25}$

For the investigation of prolonged electrochemical cycling and the associated degradation processes, it is essential to perform operando NMR measurements at higher C-rates, e.g. C/2, which are commonly used for long-term battery testing and operation. Figure 1c demonstrates the feasibility of such measurements, showing operando NMR data recorded on the same NMC811/graphite cell cycling at C/1.5. The ${ }^{7} \mathrm{Li}$ NMR spectra still exhibit the same features as observed at slow charging (Figure 1a), and the voltage profile still compares well with that of a coin cell (Figure S3b). This demonstrates that realistic electrochemical performance at practical rates can be obtained in our in situ cells while NMR measurements can still be performed for both electrodes with sufficient sensitivity and time resolution.
Temperature dependence of Li-ion mobility. Apart from cycling rates, the temperature during battery cycling also influences battery performance and lifetime, ${ }^{2,3}$ for instance by changing $\mathrm{Li}$ ion dynamics. In order to explore these effects, we performed operando NMR experiments on NMC811/graphite cells at temperatures between -5 and $+55^{\circ} \mathrm{C}$.

Figure 2a compares NMC spectra recorded during charge at 55 and $25^{\circ} \mathrm{C}$. At $55^{\circ} \mathrm{C}$, the fast decrease and subsequent recovery of the signal intensity occurs earlier during charge and leads to a more intense and sharper signal compared to the data at $25{ }^{\circ} \mathrm{C}$. Accordingly, the local minimum in the integration curve (Figure 2b) occurs after shorter charging time. This again confirms that the local minima are caused by changes in Li-ion mobility, and more precisely by a transition from the slow motion to the fast motion regime.

HIGH TEMPERATURE
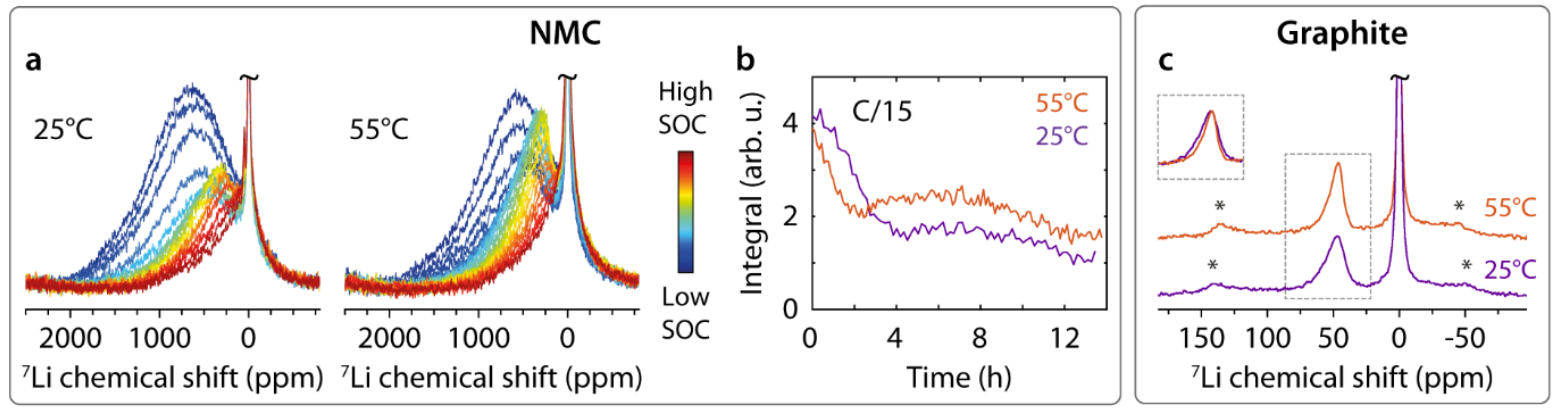

LOW TEMPERATURE
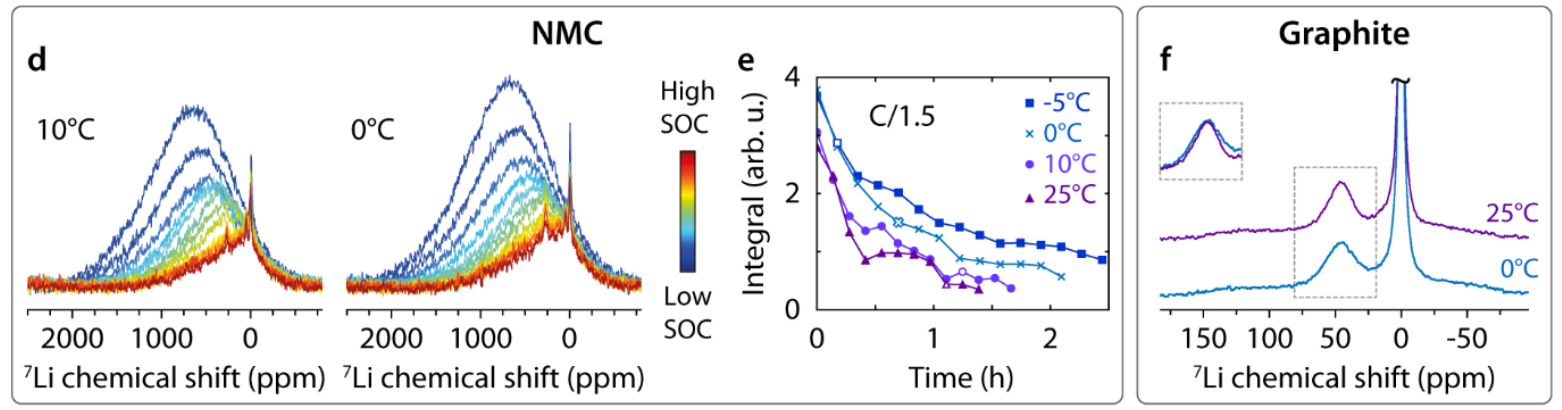

Figure 2. Li-ion mobility in NMC811 and graphite as a function of temperature, probed by operando NMR experiments on NMC811/graphite full-cells, cycled between 4.3 and 2.5 V. (a,b,d,e) ${ }^{7} \mathrm{Li}$ NMR spectra of NMC811 (a,d) and their integrated signal intensities (b,e) during charge at different temperatures. (a,b) The data was recorded at C/15 using free-standing electrode films. (d,e) The data was recorded at $\mathrm{C} / 1.5$ with a $\mathrm{CCCV}$ (constant current - constant voltage) cycling protocol using coated electrodes. The electrolyte signal was suppressed in the NMR experiments (see SI section 1 for details). An additional peak at 270 ppm appears at high SOC in (d) and is caused by Li metal. The integration plot in (e) shows curves for additional temperatures (spectra in Figure S7), and an empty symbol indicates the start of the $\mathrm{CV}$ period. (c,f) ${ }^{7} \mathrm{Li}$ NMR spectra of lithiated graphite, taken at the top of charge at different temperatures. The insets show an overlay of the dense stage peaks scaled to the same intensity, highlighting line narrowing with increasing temperature. The datasets were recorded on two different in situ cells using coated electrodes. In (c), the graphite electrode used is different from those used in the rest of this paper (see SI section 1). Due to differences in cell balancing and therefore higher lithiation of the graphite electrode in (c), the concentration of $\mathrm{LiC}_{6}$ is higher in (c) and its satellite transitions are more pronounced (marked by asterisks, see also Figure S8).

NMC spectra acquired at temperatures below $25^{\circ} \mathrm{C}$ are shown in Figures $2 \mathrm{~d}$ and S7. The cell was charged at $\mathrm{C} / 1.5$ to make efficient use of experimental time and to enable the observation of kinetically hindered processes at low temperatures. A CCCV (constant current - constant voltage) cycling protocol was applied with voltage holds at 4.3 and $2.5 \mathrm{~V}$ in order to compensate for slower kinetics. The number of points and hence resolution in the integration curves shown in Figure $2 \mathrm{e}$ is decreased at this faster charging rate, however the trends at $25^{\circ} \mathrm{C}$ compare well with those obtained at slower rates (Figure S6).
As expected, slower Li mobility is observed in NMC811 at low temperatures. At $10{ }^{\circ} \mathrm{C}$, the recovery of the ${ }^{7} \mathrm{Li}$ peak intensity caused by mobile Li ions is only barely visible (Figure $2 \mathrm{~d}$ ) and the local minimum in the integration curve (Figure 2e, circles) is less pronounced than at $25^{\circ} \mathrm{C}$ (triangles). At temperatures below $10^{\circ} \mathrm{C}$, the signal intensity decreases monotonically during charge and no local minimum occurs anymore, indicating that $\mathrm{Li}$ ions do not enter the fast motion regime at these temperatures. Moreover, the spectra at the top of charge (dark red in Figure 2d) show increased intensity and broader lineshapes as 
the temperature decreases. This indicates that more Li ions remain in NMC and cannot be extracted at $4.3 \mathrm{~V}$ anymore, reducing the available capacity. This is confirmed by comparing the charge capacities which are $190 \mathrm{mAh} / \mathrm{g}$ at $25^{\circ} \mathrm{C}$ and $168 \mathrm{mAh} / \mathrm{g}$ at $0{ }^{\circ} \mathrm{C}$.

Li-ion mobility in the graphite electrode is of particular interest at high $\mathrm{Li}$ concentration due to its role in $\mathrm{Li}$ metal deposition processes. ${ }^{14-16}$ In static ${ }^{7} \mathrm{Li}$ NMR spectra of $\mathrm{LiC}_{6}$, an increase of Li diffusivity decreases the linewidth of the central transition peak due to reduced Li-Li dipolar couplings. ${ }^{33}$ This change is detected in our operando experiments too, as demonstrated by a comparison of graphite spectra at the top of charge at different temperatures $\left(0{ }^{\circ} \mathrm{C}, 25^{\circ} \mathrm{C}, 55^{\circ} \mathrm{C}\right)$ in Figure $2 \mathrm{c}, \mathrm{f}$. Line narrowing at higher temperatures is not only observed in these spectra, but over the whole charge (Figure S9), demonstrating that these differences are indeed coming from changes of Li-ion mobility and not just from a different ratio of $\mathrm{LiC}_{6}$ and $\mathrm{LiC}_{12}$ at the top of charge. Moreover, this shows that changes of Li mobility can also be observed in this two-phase regime.

Li metal deposition at low temperatures. As outlined in the introduction, Li metal deposition on graphite is a severe degradation process in LIBs at low temperature. In ${ }^{7} \mathrm{Li}$ NMR spectra, Li metal appears at a distinct chemical shift of $\sim 260 \mathrm{ppm}$, the so-called Knight shift. ${ }^{34}$ To explore the capabilities of operando NMR for monitoring Li metal deposition and the conditions under which it occurs, we cycled three different NMC811/graphite cells (A, B, C) at different temperatures, and acquired ${ }^{7} \mathrm{Li} \mathrm{NMR}$ spectra for Li metal, NMC811 and graphite. Figure 3 shows the Li metal spectra, voltage and current profiles, and the integrated signal intensity of $\mathrm{Li}$ metal for selected cycles. All cells were cycled between 4.3 and $2.5 \mathrm{~V}$ using CCCV protocols. To facilitate analysis, yellow and orange shading in Figure 3 highlight $\mathrm{CC}$ and $\mathrm{CV}$ charge periods, respectively. The full data sets and detailed cycling protocols can be found in the SI (section 9 and Table S1).

\section{a}

Cell A

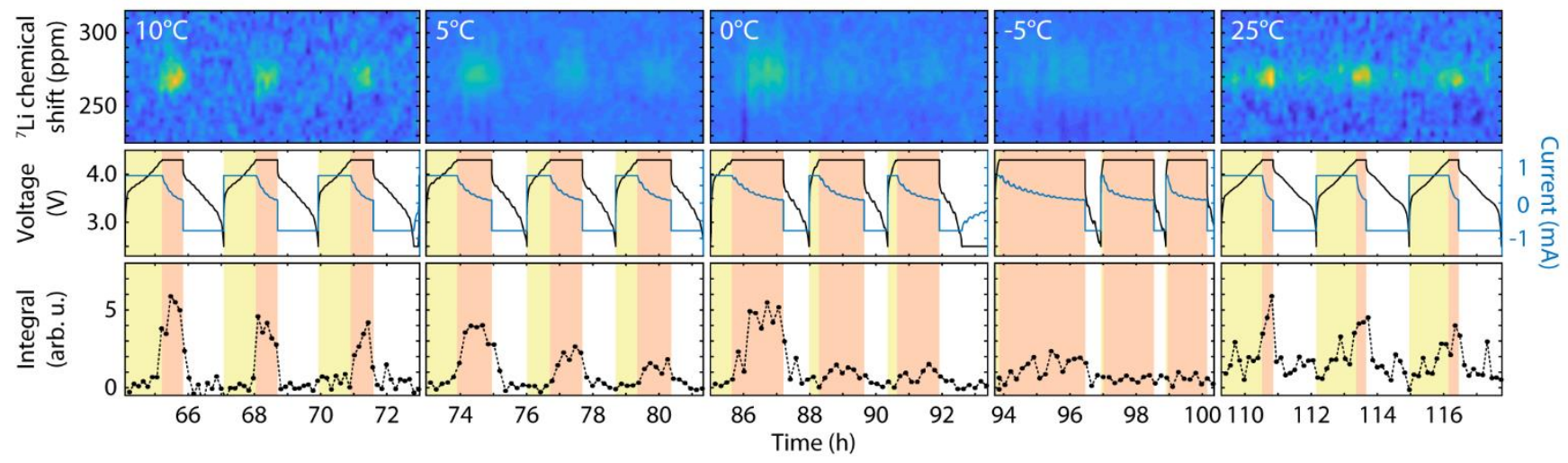

b
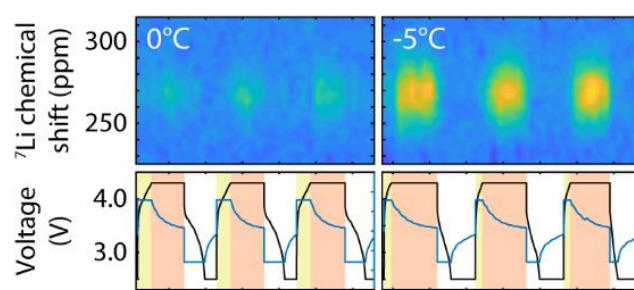

Cell B

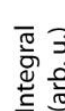

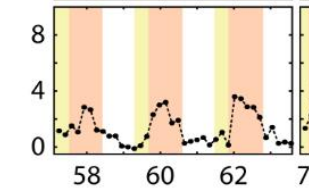

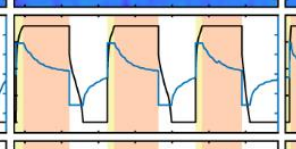

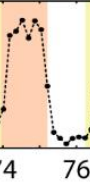

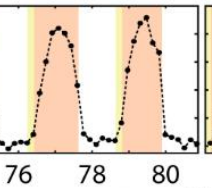
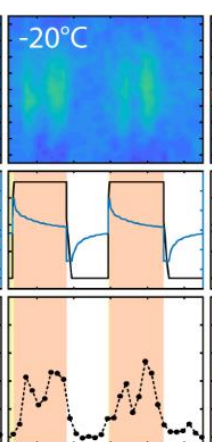
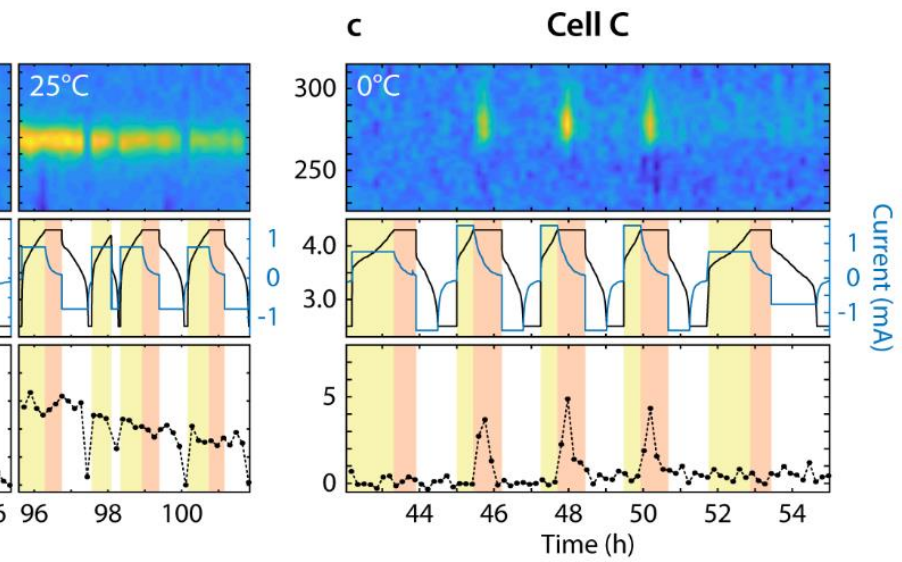

Figure 3. Operando NMR of Li metal deposition in three different NMC811/graphite full-cells assembled with coated electrodes and operating at different temperatures. The cells were cycled using CCCV protocols with voltage holds applied at $2.5 \mathrm{~V}$ (except for cell A) and $4.3 \mathrm{~V}$ until the current dropped below $\mathrm{C} / 15$. The $\mathrm{C}$-rate was $\mathrm{C} / 1.5$, except for three cycles in cell $\mathrm{C}$ at $\mathrm{C} / 0.75$. ${ }^{7} \mathrm{Li} \mathrm{NMR}$ spectra of Li metal were taken approximately every 10 minutes, alternating with NMC and graphite spectra. The top panels show the Li metal spectra and the operating temperature, the middle panels show the electrochemistry with voltage (black) and current (blue) profiles, and the bottom panels show the Li metal signal integral. Yellow and orange shading indicate CC and CV charge, respectively. For each cell, the same scaling was applied to the spectral contours and to the integrals at all temperatures, allowing an approximate comparison of the Li metal quantities (see discussion in SI section 9). The time axis corresponds to the absolute experimental time of operando NMR, meaning that, e.g., the $25^{\circ} \mathrm{C}$ spectra of cells A and B were acquired after low-temperature cycling. The full data acquired on each cell can be found in Figures S10, S11 and S13, including NMC and graphite spectra, additional cycles, and longer voltage hold periods.

While no Li metal is detected in these cells in initial room temperature experiments (see Figures S10, S11, S13), Li metal deposition does occur at low temperatures. The ${ }^{7} \mathrm{Li}$ NMR data in Figure 3 shows how Li metal deposits during charge and strips during discharge, and that no dead Li remains in the cell initially, i.e., all Li metal is removed during discharge. Moreover, Li metal deposition occurs in parallel with intercalation into graphite, and the deposits constitute not more than $\sim 2 \%$ of the 
Li transferred at the respective measurement point in most cases, with a rise up to $\sim 5 \%$ in cell $\mathrm{B}$ at $-5{ }^{\circ} \mathrm{C}$ and below (see SI, section 9). This indicates that Li metal deposition is locally restricted, a phenomenon which is also observed in commercial cells, and has been related to differences in stack pressure, temperature and potential across the cell. ${ }^{35,36} \mathrm{We}$ assume that such inhomogeneities play a role in our experiments too, as each cell is prepared individually and small differences may occur for instance in electrode alignment. This may explain the different observations for cells $\mathrm{A}$ to $\mathrm{C}$, for example regarding the temperature at which $\mathrm{Li}$ metal deposition is first detected. It is also worth noting again that we used high-quality electrodes with very good long-term performance in coin cells (Figure S3d), meaning that the effects observed here are not caused by poorlymade electrodes.

In all cells, $\mathrm{Li}$ deposition sets in at $\sim 4.3 \mathrm{~V}$, i.e. when the $\mathrm{CV}$ period is reached. Interestingly, the maximum Li metal deposition is not observed at this point where current and voltage are highest. Instead, the deposition continues at lower currents during the CV period. Similarly, in cell C, Li deposition continues at currents below $\mathrm{C} / 1.5$ after charging at $\mathrm{C} / 0.75$ while no $\mathrm{Li}$ metal is observed when charging at $\mathrm{C} / 1.5$ (before and after the $\mathrm{C} / 0.75$ cycles). Several factors may contribute to this effect. $\mathrm{Li}$ metal deposition can continue at lower currents after the initial overpotential for Li nucleation has been overcome. Moreover, the lithiation of graphite continues during the voltage hold, which means that the buildup of concentration gradients between surface and bulk continues as well if the lithiation proceeds faster than the solid diffusion. As mentioned before, further lithiation also decreases Li diffusion rates in graphite. Both effects hinder further Li intercalation and may favour Li deposition. Phase transitions in graphite may play a role, too, ${ }^{37}$ and the associated increase in overpotential as well as the drop in anode potential, e.g. at the beginning of the stage 3 to stage 2 transition, may favour Li metal deposition. Intriguingly, the strongest increase of $\mathrm{Li}$ metal signal in cell $\mathrm{B}$ at $0{ }^{\circ} \mathrm{C}$ coincides with the appearance of the $\mathrm{LiC}_{12}$ signal in the graphite spectra (Figure S12). The maximum $\mathrm{Li}$ metal quantity is mostly reached within 30 minutes of voltage hold, and a signal decrease can be observed on further hold in some cases. This can be caused by chemical intercalation into graphite and/or electrolyte degradation and SEI formation.

Interestingly, the amount of deposited Li decreases markedly when lowering the temperature to $-5^{\circ} \mathrm{C}$ in cell $\mathrm{A}$ and to $-20{ }^{\circ} \mathrm{C}$ in cell $\mathrm{B}$. This perhaps counter-intuitive observation is in line with findings by Tippmann et al. $^{38}$ and may be explained as follows. Due to the high overpotentials at these temperatures, the $\mathrm{CV}$ period is reached very quickly ( $<5 \mathrm{~min} \mathrm{CC}$ charge) and the current decreases. Therefore, the average charging current is lower and the buildup of $\mathrm{Li}$ concentration gradients in graphite may be less severe. Moreover, the capacity is reduced at lower temperature $\left(\sim 60 \mathrm{mAh} / \mathrm{g}\right.$ at $\left.-20^{\circ} \mathrm{C}\right)$ and graphite does not reach a very high $\mathrm{SOC}$, meaning that there are more $\mathrm{Li}$ vacancies in the material, the solid diffusion limitations may be smaller and the overpotential for Li plating higher.

Reduced Li deposition is also observed when no voltage hold periods at $2.5 \mathrm{~V}$ are applied (cell $\mathrm{A}$ ), even though the upper cutoff potential still lies at $4.3 \mathrm{~V}$. As the charge starts at a comparably high $\mathrm{SOC}$ in these cases, the charging times and in particular the CC period are reduced and again Li concentration gradients in the graphite particles may be less pronounced. This means that reduced charging times may generally help in reducing Li deposition even when charging to a relatively high voltage, which could be implemented in pulse charge-type protocols alternating CC charge with rest periods.

When returning to room temperature after low temperature cycling, we observe clear consequences of the previous Li metal deposition. In cell $\mathrm{B}$, a persistent $\mathrm{Li}$ metal signal can be identified which is hardly affected by charge and discharge. It can therefore be attributed to dead $\mathrm{Li}$, formed during additional lowtemperature cycles (Figure S11). The slow overall signal decay is presumably caused by reaction with the electrolyte, and the disappearance of the signal at full discharge can be attributed to line broadening effects induced by strong anisotropic bulk magnetic susceptibility effects in delithiated graphite. ${ }^{39}$ A more interesting observation - which has, to our knowledge, not been reported before - is made in cell $\mathrm{A}$ where Li metal plating and stripping now also takes place at $25^{\circ} \mathrm{C}$. This is most likely a consequence of increased SEI buildup and the blocking of graphite pores in areas where Li metal was formed at low temperature. ${ }^{40}$ These observations highlight that $\mathrm{Li}$ deposition at low temperature will continue to accelerate capacity fade even under previously "safe" operating conditions and that it should therefore be avoided as much as possible.

Finally, it is worth noting that no indications of Li deposition are observed in the voltage curves. ${ }^{41}$ This conforms with the general notion that local or minor deposition can often not be detected due to averaging effects over the whole cell, particularly at rates used in practical cells. ${ }^{16}$ Moreover, no sign of Li metal deposition was seen by scanning electron microscopy (Figure S14). These observations further highlight the strength of operando NMR in revealing the presence of small but detrimental quantities of Li metal. Overall, the occurrence and extent of Li metal deposition under different charging conditions is a complex problem, involving the interplay of multiple processes in different parts of the cell, on which our experiments offer valuable insights.

Electrodes with kinetic limitations. We performed initial operando NMR experiments on NMC811/graphite cells using free-standing electrode films. They were made from the same active materials as the coated electrodes but contained more than twice as much active material and were considerably thicker. It is instructive to examine the operando NMR data acquired with these electrodes, since they provide a method for exploring the effects of kinetic limitations on $\mathrm{Li}$ (de)intercalation and Li metal deposition.

The spectra acquired on a NMC811/graphite cell with freestanding electrodes are shown in Figure 4a, along with the signal integration in Figure $4 \mathrm{~b}$. As expected, the spectra recorded at $\mathrm{C} / 14$ resemble those in Figure 1a. However, the cell performance deteriorates considerably at higher rates: the discharge capacity drops from $195 \mathrm{mAh} / \mathrm{g}$ at C/14 to $127 \mathrm{mAh} / \mathrm{g}$ at C/3.5. Accordingly, the NMR spectra at $\mathrm{C} / 3.5$ show that graphite and NMC are not fully lithiated anymore at high and low SOC, respectively (see integrals at $55 \mathrm{~h}<t<68 \mathrm{~h}$ ). The full capacity of $195 \mathrm{mAh} / \mathrm{g}$ can be recovered by applying CV periods at 2.5 and $4.3 \mathrm{~V}$ as demonstrated in the last cycle in Figure 4, indicating that capacity loss is caused by kinetic limitations. This is further supported by the fact that $\mathrm{Li}$ metal deposition is observed under such mild charging conditions $\left(25^{\circ} \mathrm{C}, \mathrm{C} / 7\right)$, again causing an additional signal at $\sim 260 \mathrm{ppm}$ (NMC spectra in Figure $4 \mathrm{a}$ ). 

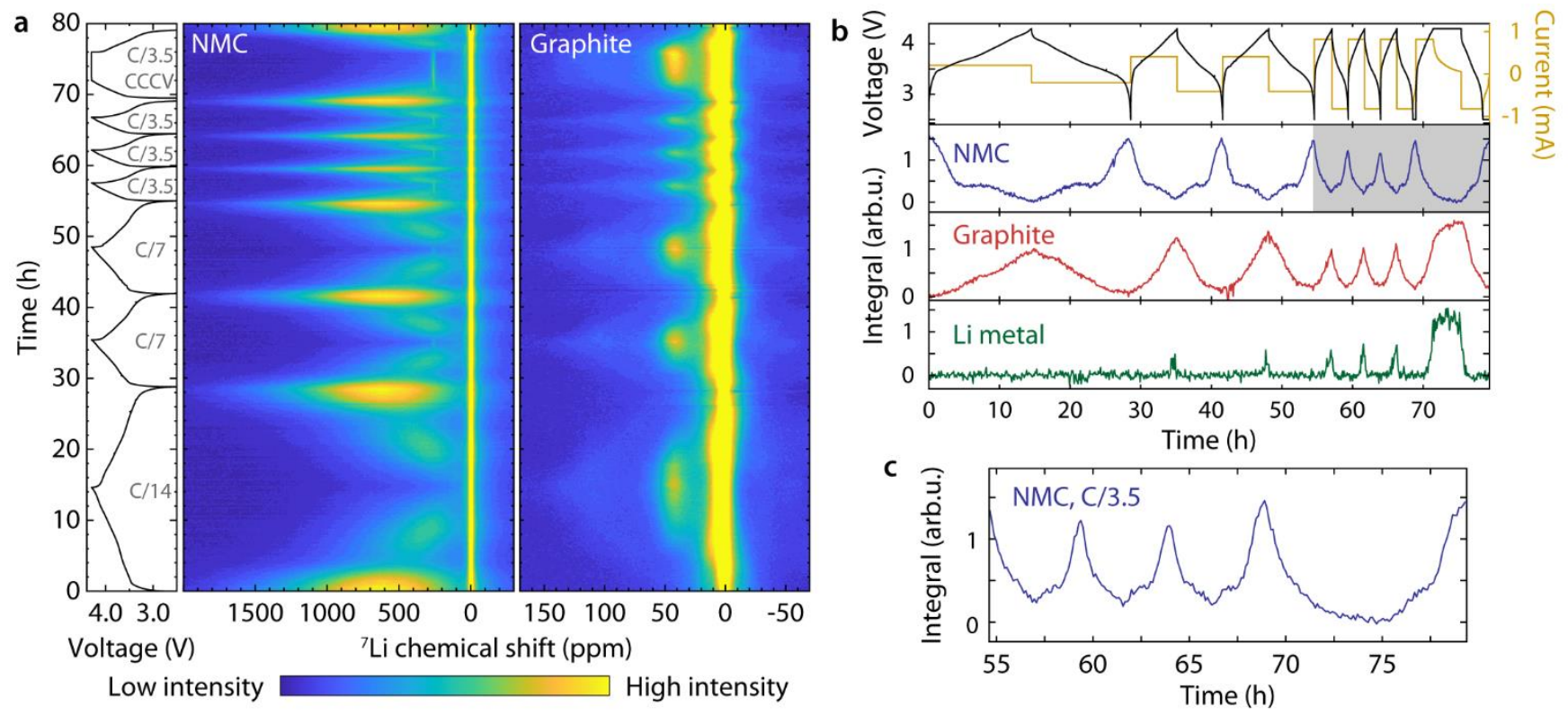

Figure 4. (a) Operando ${ }^{7} \mathrm{Li}$ NMR data acquired on a NMC811/graphite full-cell made with free-standing electrode films, cycled between 4.3 and $2.5 \mathrm{~V}$ at $25^{\circ} \mathrm{C}$ and different C-rates as indicated in the Figure. (b) Voltage and current profiles together with integrated signal intensities of NMC, graphite and Li metal. The Li metal integral was obtained from its signal in the graphite spectra (not shown). All integrals are shown with arbitrary scaling. (c) Zoom of the grey shaded region in (b), showing the NMC integrals at C/3.5.

Kinetic limitations in these electrodes can originate from poor electrical connectivity between active material particles, hindered (tortuous) Li-ion transport within the electrodes, ${ }^{42}$ and higher contact resistance due to the absence of metal current collector foils. Beyond the effects outlined above, this can lead to reaction heterogeneity within the electrodes as some particles are more electrochemically reactive than others. ${ }^{43}$

This becomes visible in the voltage and differential capacity profiles (Figure S3a-c) as well as in the NMC spectra: at $\mathrm{C} / 3.5$, the local minimum in the integration curve associated with the onset of fast Li-ion mobility is no longer observed during charge, but still occurs during discharge (Figure 4c, most clearly during the CCCV cycle). This may be explained as follows (see also Figure S15 for a cartoon illustrating the processes). During charge, the more reactive particles are delithiated first which increases Li-ion mobility in these particles. This then facilitates their further extraction, delaying the delithiation of less reactive particles. Due to this charge heterogeneity, no clear signs of mobile Li ions are detected. During discharge on the other hand, re-intercalated Li ions diffuse fast within all NMC particles. When approaching low SOC, the insertion of more Li ions would decrease diffusion, which is why it may become more favourable to lithiate the less reactive particles with high Li diffusivity first. Overall, this leads to a smaller discrepancy in SOC and hence a more distinguishable local minimum in the signal integration during discharge. Note that such reaction heterogeneity may also lead to overcharge of the more reactive particles, which will accelerate surface reactions and degradation. $^{3}$

Overall, the rate performance in this cell with free-standing films is poor, leading to Li metal deposition even at room temperature and relatively slow rates, along with reaction heterogeneity. The performance could be improved by optimizing the electrode preparation, but the use of coated electrodes as employed in the other experiments in this paper should clearly be preferred for measurements at higher rates.

\section{CONCLUSION}

We have presented operando NMR studies of NMC811/graphite full-cells made with industry-grade electrodes, showing realistic and reproducible cycling performance at charging rates up to at least $\mathrm{C} / 1.5$. The ${ }^{7} \mathrm{Li} \mathrm{NMR}$ signals of $\mathrm{Li}$ ions in both electrodes were recorded during electrochemical cycling and separated based on their different $T_{1}$ relaxation times. Measurements over a wide range of temperatures and rates enabled us to analyse intercalation processes in both electrodes, changes of Li-ion mobility, and Li metal deposition on graphite at low temperature.

In NMC811, a rapid loss and subsequent recovery of the ${ }^{7} \mathrm{Li}$ NMR signal, i.e. a local minimum in the integration-vs-time plot, indicates a transition from slow to fast Li-ion mobility during charge which agrees well with our previous ex situ MAS NMR studies. ${ }^{25} \mathrm{Li}$-ion mobility is reduced with decreasing temperature, as seen via the shift of the local minimum to higher SOC and its eventual disappearance. Temperature-dependent changes of Li-ion mobility in dense stage graphite are also shown by the operando NMR data where increasing Li mobility leads to line narrowing.

The real-time detection of Li metal deposition on graphite is an intriguing capability of NMR, and a task with which many other analytical techniques struggle considerably. ${ }^{16}$ Even small quantities of Li metal can be detected, which do not necessarily cause electrochemical features but still contribute to cell degradation. In our experiments with a $\mathrm{CC}$ charge and a subsequent $\mathrm{CV}$ period at $4.3 \mathrm{~V}, \mathrm{Li}$ deposition starts at around $4.3 \mathrm{~V}$ - well before the full lithiation of graphite - and surprisingly continues even at lower currents during the voltage hold. Hence, reducing the current may not be sufficient if $\mathrm{Li}$ deposition has commenced already, and long CV charges can be harmful in such cases. On the other hand, the length of the $\mathrm{CC}$ charge period also appears to play a crucial role. Shorter CC periods to the same upper cutoff voltage lead to less Li deposition, as observed at very low temperature, but also when charging from a higher 
SOC. This suggests that not only lower charging currents, but also shorter charging periods may reduce Li deposition. Finally, we have shown the effect of Li deposition on subsequent room temperature cycling where we not only observe dead Li but also fresh Li deposition under these mild charging conditions. This shows the continued detrimental effects of low-temperature $\mathrm{Li}$ deposition, and how crucial it is to avoid this process.

For comparison to the results obtained on well-performing cells, we have also presented operando NMR data on a NMC811/graphite cell with thick free-standing electrode films. This cell showed poor rate performance, and the NMR data revealed Li metal deposition under mild charging conditions and reaction heterogeneity in the NMC electrode. This highlights the necessity of using well-prepared cells and electrodes for studying electrochemical cycling at high charging rates.

Overall, the experiments presented here show that operando NMR is a very powerful tool for observing multiple processes in parallel during electrochemical cycling of full-cells. This includes phenomena that concern the complete electrode, but also minor processes which cannot be detected by electrochemical measurements alone and may also be missed in ex situ studies of a relaxed and/or discharged cell. Lastly, the realistic cycling performance of our in situ cells means that the conclusions drawn here can be expected to apply to other NMC811/graphite cell types as well. We anticipate this NMR methodology and the separate observation of different cell compartments to be transferrable to other LIB systems, and to provide further valuable insights into different aspects of battery cycling.

\section{ASSOCIATED CONTENT}

Supporting Information. Details of additional electrodes, in situ NMR cell assembly, additional NMR details, overview of operando NMR cells, electrochemical cycling data, discussion of increasing probe sensitivity, comparison of 1D spectra during first and second charge, comparison of NMC integrals at different charging rates, additional NMC spectra at different temperatures, comparison of $\mathrm{LiC}_{12} / \mathrm{LiC}_{6}$ spectra of different electrodes, linewidth of $\mathrm{LiC}_{12} / \mathrm{LiC}_{6}$ peak during charge, additional operando $\mathrm{NMR}$ data of Li metal deposition, SEM of graphite electrodes, charge heterogeneity in cells with free-standing electrodes, influence of in situ cell orientation on ${ }^{7} \mathrm{Li} \mathrm{NMR}$ spectra, operando NMR data of a NMC811/Li half-cell. This material is available free of charge via the Internet at http://pubs.acs.org.

\section{AUTHOR INFORMATION}

\section{Corresponding Author}

*cpg27@cam.ac.uk

\section{Author Contributions}

The manuscript was written through contributions of all authors. All authors have given approval to the final version of the manuscript.

\section{ACKNOWLEDGMENT}

This work was supported by the Faraday Institution under grant number FIRG001. KM thanks Dylan Maxwell, Yuning Zhou, Anna Gunnarsdóttir and Dr. Lina Zhou for helpful discussions and support with initial operando NMR experiments and sample preparation.

\section{REFERENCES}

(1) Tarascon, J.-M.; Armand, M. Issues and Challenges Facing Rechargeable Lithium Batteries. Nature 2001, 414 (6861), 359-

367.

Vetter, J.; Novák, P.; Wagner, M. R.; Veit, C.; Möller, K.-C.; Besenhard, J. O.; Winter, M.; Wohlfahrt-Mehrens, M.; Vogler, C.; Hammouche, A. Ageing Mechanisms in Lithium-Ion Batteries. J. Power Sources 2005, 147 (1-2), 269-281.

Palacín, M. R. Understanding Ageing in Li-Ion Batteries: A Chemical Issue. Chem. Soc. Rev. 2018, 47 (13), 4924-4933.

Harks, P. P. R. M. L.; Mulder, F. M.; Notten, P. H. L. In Situ Methods for Li-Ion Battery Research: A Review of Recent Developments. J. Power Sources 2015, 288, 92-105.

Pecher, O.; Carretero-González, J.; Griffith, K. J.; Grey, C. P. Materials' Methods: NMR in Battery Research. Chem. Mater. 2017, 29 (1), 213-242.

Kayser, S. A.; Mester, A.; Mertens, A.; Jakes, P.; Eichel, R.-A.; Granwehr, J. Long-Run in Operando NMR to Investigate the Evolution and Degradation of Battery Cells. Phys. Chem. Chem. Phys. 2018, 20 (20), 13765-13776.

Sorte, E. G.; Banek, N. A.; Wagner, M. J.; Alam, T. M.; Tong, Y J. In Situ Stripline Electrochemical NMR for Batteries. ChemElectroChem 2018, 5 (17), 2336-2340.

Freytag, A. I.; Pauric, A. D.; Krachkovskiy, S. A.; Goward, G. R. In Situ Magic-Angle Spinning ${ }^{7} \mathrm{Li}$ NMR Analysis of a Full Electrochemical Lithium-Ion Battery Using a Jelly Roll Cell Design. J. Am. Chem. Soc. 2019, 141 (35), 13758-13761.

Kim, J.; Lee, H.; Cha, H.; Yoon, M.; Park, M.; Cho, J. Prospect and Reality of Ni-Rich Cathode for Commercialization. Adv. Energy Mater. 2018, 8 (6), 1702028.

Wang, X.; Ding, Y.; Deng, Y.; Chen, Z. Ni-Rich/Co-Poor Layered Cathode for Automotive Li-Ion Batteries: Promises and Challenges. Adv. Energy Mater. 2020, 10 (12), 1903864.

Noh, H.-J.; Youn, S.; Yoon, C. S.; Sun, Y.-K. Comparison of the Structural and Electrochemical Properties of Layered $\mathrm{Li}\left[\mathrm{Ni}_{\mathrm{x}} \mathrm{Co}_{\mathrm{y}} \mathrm{Mn}_{\mathrm{z}}\right] \mathrm{O}_{2}(\mathrm{x}=1 / 3,0.5,0.6,0.7,0.8$ and 0.85$)$ Cathode Material for Lithium-Ion Batteries. J. Power Sources 2013, 233, 121-130.

Persson, K.; Sethuraman, V. A.; Hardwick, L. J.; Hinuma, Y.; Meng, Y. S.; van der Ven, A.; Srinivasan, V.; Kostecki, R.; Ceder, G. Lithium Diffusion in Graphitic Carbon. J. Phys. Chem. Lett. 2010, 1 (8), 1176-1180.

Van der Ven, A.; Bhattacharya, J.; Belak, A. A. Understanding Li Diffusion in Li-Intercalation Compounds. Acc. Chem. Res. 2013, 46 (5), 1216-1225.

Li, Z.; Huang, J.; Yann Liaw, B.; Metzler, V ; Zhang, J. A Review of Lithium Deposition in Lithium-Ion and Lithium Metal Secondary Batteries. J. Power Sources 2014, 254, 168-182.

Liu, Q.; Du, C.; Shen, B.; Zuo, P.; Cheng, X.; Ma, Y.; Yin, G.; Gao, Y. Understanding Undesirable Anode Lithium Plating Issues in Lithium-Ion Batteries. RSC Adv. 2016, 6 (91), 88683 88700.

Waldmann, T.; Hogg, B.-I.; Wohlfahrt-Mehrens, M. Li Plating as Unwanted Side Reaction in Commercial Li-Ion Cells - A Review. J. Power Sources 2018, 384, 107-124.

Hasan, M. F.; Chen, C.-F.; Shaffer, C. E.; Mukherjee, P. P. Analysis of the Implications of Rapid Charging on Lithium-Ion Battery Performance. J. Electrochem. Soc. 2015, 162 (7), A1382A1395.

Bhattacharyya, R.; Key, B.; Chen, H.; Best, A. S.; Hollenkamp, A. F.; Grey, C. P. In Situ NMR Observation of the Formation of Metallic Lithium Microstructures in Lithium Batteries. Nat. Mater. 2010, 9 (6), 504-510.

Küpers, V.; Kolek, M.; Bieker, P.; Winter, M.; Brunklaus, G. In Situ 7 Li-NMR Analysis of Lithium Metal Surface Deposits with Varying Electrolyte Compositions and Concentrations. Phys. Chem. Chem. Phys. 2019, 21 (47), 26084-26094.

Gunnarsdóttir, A. B.; Vema, S.; Menkin, S.; Marbella, L. E.; Grey, C. P. Investigating the Effect of a Fluoroethylene Carbonate Additive on Lithium Deposition and the Solid Electrolyte Interphase in Lithium Metal Batteries Using in Situ NMR Spectroscopy. J. Mater. Chem. A 2020, 8 (30), 1497514992.

Wandt, J.; Jakes, P.; Granwehr, J.; Eichel, R.-A.; Gasteiger, H. A. Quantitative and Time-Resolved Detection of Lithium Plating on Graphite Anodes in Lithium Ion Batteries. Mater. Today 2018, 21 (3), 231-240.

Arai, J.; Nakahigashi, R. Study of Li Metal Deposition in Lithium 
Ion Battery during Low-Temperature Cycle Using In Situ SolidState ${ }^{7} \mathrm{Li}$ Nuclear Magnetic Resonance. J. Electrochem. Soc. 2017, 164 (13), A3403-A3409.

(23) Pecher, O.; Bayley, P. M.; Liu, H.; Liu, Z.; Trease, N. M.; Grey C. P. Automatic Tuning Matching Cycler (ATMC) in Situ NMR Spectroscopy as a Novel Approach for Real-Time Investigations of Li- and Na-Ion Batteries. J. Magn. Reson. 2016, 265, 200-209. Massiot, D.; Farnan, I.; Gautier, N.; Trumeau, D.; Trokiner, A.; Coutures, J. P. ${ }^{71} \mathrm{Ga}$ and ${ }^{69} \mathrm{Ga}$ Nuclear Magnetic Resonance Study of $\beta-\mathrm{Ga}_{2} \mathrm{O}_{3}$ : Resolution of Four- and Six-Fold Coordinated Ga Sites in Static Conditions. Solid State Nucl. Magn. Reson. 1995, 4 (4), 241-248.

(25) Märker, K.; Reeves, P. J.; Xu, C.; Griffith, K. J.; Grey, C. P. Evolution of Structure and Lithium Dynamics in $\mathrm{LiNi}_{0.8} \mathrm{Mn}_{0.1} \mathrm{Co}_{0.1} \mathrm{O}_{2}$ (NMC811) Cathodes during Electrochemical Cycling. Chem. Mater. 2019, 31 (7), 2545-2554.

(26) Lorie Lopez, J. L.; Grandinetti, P. J.; Co, A. C. Enhancing the Real-Time Detection of Phase Changes in Lithium-Graphite Intercalated Compounds through Derivative Operando (DOp) NMR Cyclic Voltammetry. J. Mater. Chem. A 2018, 6 (1), 231243.

(27) Grey, C. P.; Dupré, N. NMR Studies of Cathode Materials for Lithium-Ion Rechargeable Batteries. Chem. Rev. 2004, 104 (10), 4493-4512.

(28) Guerard, D.; Herold, A. Intercalation of Lithium into Graphite and Other Carbons. Carbon N. Y. 1975, 13 (4), 337-345.

(29) Dahn, J. R. Phase Diagram of $\mathrm{Li}_{\mathrm{x}} \mathrm{C}_{6}$. Phys. Rev. B 1991, 44 (17), 9170-9177.

(30) Letellier, M.; Chevallier, F.; Béguin, F. In Situ ${ }^{7}$ Li NMR during Lithium Electrochemical Insertion into Graphite and a Carbon/Carbon Composite. J. Phys. Chem. Solids 2006, 67 (56), 1228-1232.

(31) Letellier, M.; Chevallier, F.; Morcrette, M. In Situ ${ }^{7}$ Li Nuclear Magnetic Resonance Observation of the Electrochemical Intercalation of Lithium in Graphite; 1st Cycle. Carbon N. Y. 2007, 45 (5), 1025-1034.

(32) Zhou, L.; Leskes, M.; Liu, T.; Grey, C. P. Probing Dynamic Processes in Lithium-Ion Batteries by In Situ NMR Spectroscopy: Application to $\mathrm{Li}_{1.08} \mathrm{Mn}_{1.92} \mathrm{O}_{4}$ Electrodes. Angew. Chemie Int. Ed. 2015, 54 (49), 14782-14786.

(33) Langer, J.; Epp, V.; Heitjans, P.; Mautner, F. A.; Wilkening, M. Lithium Motion in the Anode Material $\mathrm{LiC}_{6}$ as Seen via Time-
Domain ${ }^{7} \mathrm{Li}$ NMR. Phys. Rev, B 2013, 88 (9), 094304

Gutowsky, H. S.; McGarvey, B. R. Nuclear Magnetic Resonance in Metals. I. Broadening of Absorption Lines by Spin-Lattice Interactions. J. Chem. Phys. 1952, 20 (9), 1472-1477.

Bach, T. C.; Schuster, S. F.; Fleder, E.; Müller, J.; Brand, M. J Lorrmann, H.; Jossen, A.; Sextl, G. Nonlinear Aging of Cylindrical Lithium-Ion Cells Linked to Heterogeneous Compression. J. Energy Storage 2016, 5, 212-223.

Ng, B.; Coman, P. T.; Faegh, E.; Peng, X.; Karakalos, S. G.; Jin, X.; Mustain, W. E.; White, R. E. Low-Temperature Lithium Plating/Corrosion Hazard in Lithium-Ion Batteries: Electrode Rippling, Variable States of Charge, and Thermal and Nonthermal Runaway. ACS Appl. Energy Mater. 2020, 3 (4), 3653-3664.

Heß, M.; Novák, P. Shrinking Annuli Mechanism and StageDependent Rate Capability of Thin-Layer Graphite Electrodes for Lithium-Ion Batteries. Electrochim. Acta 2013, 106, 149-158.

Tippmann, S.; Walper, D.; Balboa, L.; Spier, B.; Bessler, W. G. Low-Temperature Charging of Lithium-Ion Cells Part I: Electrochemical Modeling and Experimental Investigation of Degradation Behavior. J. Power Sources 2014, 252, 305-316.

Hiroyama, Y.; Kume, K. High Resolution ${ }^{13} \mathrm{C}$ NMR Spectra in Graphite Chemical Shift and Diamagnetism. Solid State Commun. 1988, 65 (7), 617-619.

Yang, X.-G.; Leng, Y.; Zhang, G.; Ge, S.; Wang, C.-Y. Modeling of Lithium Plating Induced Aging of Lithium-Ion Batteries: Transition from Linear to Nonlinear Aging. J. Power Sources 2017, 360, 28-40.

Bugga, R. V.; Smart, M. C. Lithium Plating Behavior in LithiumIon Cells. In ECS Transactions; ECS, 2010; Vol. 25, pp 241-252.

Gao, H.; Wu, Q.; Hu, Y.; Zheng, J. P.; Amine, K.; Chen, Z. Revealing the Rate-Limiting Li-Ion Diffusion Pathway in Ultrathick Electrodes for Li-Ion Batteries. J. Phys. Chem. Lett. 2018, 9 (17), 5100-5104.

Liu, H.; Kazemiabnavi, S.; Grenier, A.; Vaughan, G.; Di Michiel, M.; Polzin, B. J.; Thornton, K.; Chapman, K. W.; Chupas, P. J. Quantifying Reaction and Rate Heterogeneity in Battery Electrodes in 3D through Operando X-Ray Diffraction Computed Tomography. ACS Appl. Mater. Interfaces 2019, 11 (20), 1838618394. 
Table of Contents artwork:

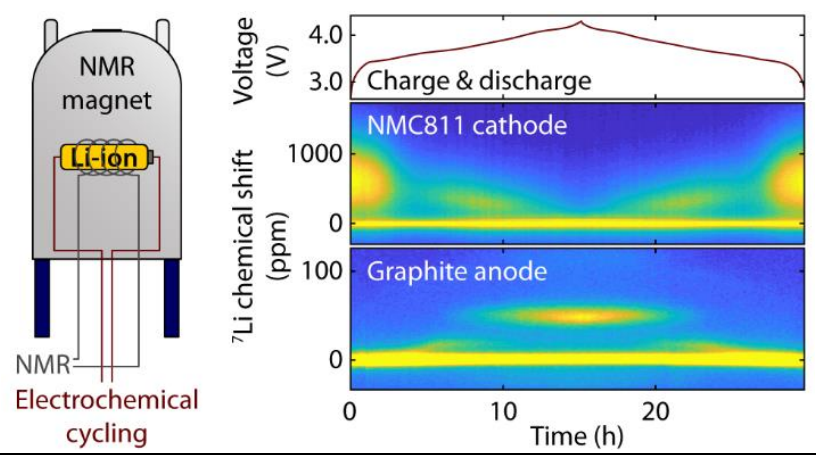

